\title{
Knowledge, Attitude and Practice of Health Care Ethics Among Medical, Dental and Nursing Colleges Students and Faculty in Visakhapatnam: A Questionnaire Study
}

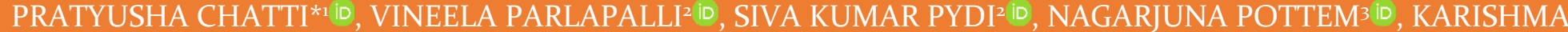
JANAPAREDDY'ID, ADITHYA TEJA PRASAD PALLEKONDA'

BACKGROUND: Dental ethics revolves around the extent to which actions within the dental practice promote good and reduce harm.

AIM: The study aims to assess the knowledge, attitude, and practice among the medical, dental, and nursing college students and faculty Visakhapatnam.

MATERIALS AND METHODS: The 26-item questionnaire, self-administered, semi-structured, was designed to access the student's and faculty's knowledge, attitudes, and practices in the globe of healthcare ethics. With all the exclusion and inclusion criteria, the total sample derived as 1190. Statistical analysis was done using the SPSS Software Version 21.0 with the significant p-value at $\leq 0.05$. Descriptive statistics, chi-Square test and ANOVA test used to compare the knowledge attitude and practice of dental, medical, and nursing students and faculty. RESULTS: Out of 1,190 participants, 456 participants belonged to medical out of which 239 are females, and 217 are males, 424 participants are from the specialty dental, 248 are females, and 176 are 310 participants belong to nursing in which 230 females and 80 males. The knowledge and attitude of dental college interns and postgraduates showed no significant difference in the Hippocratic oath, Nuremberg code, ICMR guidelines, Helsinki declaration with p-value $0.971,0.899,0.506$, and 0.768 . The knowledge and attitude of medical college interns and postgraduates showed a significant difference in the Hippocratic oath, Nuremberg code, ICMR guidelines with p-values o.oo2, 0.040 and 0.011 , respectively. The knowledge and attitude of final years showed considerable differences from the third years of nursing. CONCLUSION: The knowledge and attitude of health care ethics are significantly more in medical postgraduates than the medical interns, showed no significant difference in dental interns and graduates, and showed significant differences in nursing students.

KEYWORDS: Ethics, Knowledge, Attitude, Questionnaire, Students, Faculty

\section{INTRODUCTION}

Bioethics is a relatively new subspecialty in the medical field. It is still in its infancy in many parts of the world. ${ }^{1}$ Healthcare ethics is not routinely taught to medical professionals. There are reports that even the word "ethics" has completely ignored during the undergraduate medical curriculum. ${ }^{2}$ The same may be right with other healthcare providers' training, such as nursing professionals and other paramedical professionals. Hence it is not surprising that the theory and application of healthcare ethics in day-to-day practice are still unknown to many healthcare providers. In such a situation, the practice of ethics in healthcare will very much be influenced by the cultural background and beliefs of the people in every region. ${ }^{3}$

Ethics is a generic term referring to the ethicalness in civilization and the rules, customs, and beliefs of that society. It agreed that 'morality' is all about right and wrong based on socially approved norms of human conduct. From childhood, we learn moral rules along with other social practices. Later in life, we can distinguish between general social rules held by all members of society, and specific social regulations or ethical guidelines binding the members of particular groups such as a health care profession. ${ }^{3}$ Healthcare ethics deals with how providers apply a moral code of conduct to patients in a healthcare setting, taking into account the patients' self-respect, individuality, safety, and welfare. Clinical ethics refers to the ethics of activity in the clinical context, which, if practiced, will lead to more ethical care. For a clinical crew to practice clinical ethics, their clinical expertise and subject training need to be honed by appropriate clinical ethics training. ${ }^{4}$ Therefore, they are expected to know ethical principles and apply them in their practice. There are reports of malicious tactics patterns of medical students and medical and dental practitioners with patients as well as colleagues. Reports have stressed the need to incorporate ethical and legal issues into their curricula. Further, it has observed that health training in India provides little guidance for these professionals to resolve the ethical dilemmas they encounter.,

With this background, the present study aimed 
to enlighten the knowledge, attitude, and practice regarding health care ethics among medical, dental, and nursing students and faculty in Visakhapatnam. Andhra Pradesh.

\section{MATERIALS AND METHOD}

Study Design: An observational study was conducted using a pre-tested and pre-validated questionnaire among the students and faculty of four medical colleges, two dental colleges, and three nursing colleges in Visakhapatnam.

Ethical clearance: The study design was reviewed and approved by the Institutional Ethical Committee. The study's permission for the conduct was obtained from the concerned authorities of all the participating institutions, and written informed consent obtained from the study subjects after explaining them the purpose and methodology of the study.

Data Collection: The questionnaire was adopted from Chandrashekar et al. and Hariharan et al. and modified accordingly. ${ }^{7,10}$ The 26 -item questionnaire was a selfadministrated, semi-structured one with both open and close-ended questions accomplish to appraise the student's and faculty's knowledge, attitudes, and practices in the globe of healthcare ethics. The demographic variables included were the year of the study, age and gender, and specialty.

Inclusion \& Exclusion criteria: The participants studying in respected professional colleges around Visakhapatnam and those holding a degree of MS/MD/MDS with being in the teaching profession and those who are willing to participate in the study and present on that day of the study were included in the study. The participants who did not consent to participate were excluded from the study.

Study Participants: A total number of 1200 sample were approached (convenience sampling), in which 456 participants were from the medical speciality; 424 participants were from dental speciality, and 310 belonged to the nursing speciality. With all the exclusion and intrusion criteria, the final sample derived as 1190.

Statistical Analysis: The statistical analysis was done by the SPSS Version 21.0, and the $\mathrm{p}$-value set at $\mathrm{p} \geq 0.05$. Descriptive statistics were applied. Comparison of knowledge, attitude, and practice with dental and medical and nursing students was done by chi-square test while the comparison of knowledge, attitude, and practice with dental; medical and nursing faculty through one-way ANOVA.

\section{RESULTS}

In table 1 Out of 1,190 participants 456 were in the medical specialty in which $320(70.2 \%)$ were under graduates and $64(14.0 \%)$ were post graduates and $72(15.7 \%)$ were faculty; $217(47.5 \%)$ were males and 239(52.4\%) were females. Among 424 participants in dental specialty, $270(63.4 \%)$ were undergraduates; $8 \mathrm{o}(18.8 \%)$ were post graduates and $74(17.4 \%)$ were faculty; $176(41.5 \%)$ were males and 248 (58.4\%) were females. 310 were in specialty of nursing in which $155(50.0 \%)$ were in final year and $125(40.3 \%)$ were in third year and $30(9.7 \%)$ were faculties. The mean age of all the participants were $27.5 \pm 8.5$ years.

\begin{tabular}{|c|c|c|c|c|}
\hline \multicolumn{2}{|c|}{ Demographic data } & $\begin{array}{l}\text { Dental } \\
(\mathrm{n}=424) \\
(\%)\end{array}$ & $\begin{array}{l}\text { Medical } \\
(\mathrm{n}=456) \\
(\%)\end{array}$ & $\begin{array}{l}\text { Nursing } \\
(\mathrm{n}=\mathbf{3 1 0}) \\
(\%)\end{array}$ \\
\hline \multicolumn{2}{|c|}{ Mean Age } & \multicolumn{3}{|l|}{$27.5 \pm 8.5$} \\
\hline \multirow[b]{2}{*}{ Gender } & Males & $176(41.5 \%)$ & $217(47.5 \%)$ & $80(25.8 \%)$ \\
\hline & Females & $\begin{array}{l}248 \\
(58.4 \%)\end{array}$ & $239(52.4 \%)$ & $230(74.1 \%)$ \\
\hline \multirow{3}{*}{$\begin{array}{l}\text { Year of } \\
\text { study }\end{array}$} & $\begin{array}{l}\text { Interns/final } \\
\text { years/third } \\
\text { years }\end{array}$ & $270(63.4 \%)$ & $320(70.2 \%)$ & $\begin{array}{l}155(50.0 \%) \\
125(40.3 \%)\end{array}$ \\
\hline & PG's & $8 \mathrm{o}(18.8 \%)$ & $64(14.0 \%)$ & -- \\
\hline & $\begin{array}{l}\text { Teaching } \\
\text { Staff }\end{array}$ & $74(17.4 \%)$ & $72(15 \cdot 7 \%)$ & 30(9.7\%) \\
\hline
\end{tabular}

Table 1. Geographic Distribution of the Study Population

Table 2 describes knowledge; attitude and practice of the studied population in which dental postgraduates and interns had no significant difference in the knowledge regarding Hippocratic oath ( $>>0.971)$; Nuremberg code ( $>0.899)$; ICMR and Helsinki declaration ( $p>0.768)$. Most of the dental postgraduates had the attitude that they had better knowledge than patients ( $p>0.654)$ and there was significant difference between practice scores of dental postgraduates with comparison of dental interns ( $>>0.02)$ and most of dental postgraduates and interns have a practice of approaching the colleges for health care problem.

It was observed that medical postgraduates and interns had statistical difference in knowledge about the Hippocratic oath $(\mathrm{p}<0.002)$; Nuremberg code $(\mathrm{p}<0.04) ; \quad$ ICMR guidelines $\quad(\mathrm{p}<0.011) ; \quad$ Helsinki 


\begin{tabular}{|c|c|c|c|c|c|c|c|c|c|c|c|c|c|}
\hline QUESTION & DI & DP & $\mathbf{p V}$ & MCI & MCPG & $\mathbf{p V}$ & NTY & NFY & $\mathbf{p V}$ & MF & DF & NF & $\mathbf{p V}$ \\
\hline \multicolumn{14}{|c|}{ KNOWLEDGE } \\
\hline $\begin{array}{c}\text { Do you know about } \\
\text { Hippocratic oath? } \\
\text { Yes } \\
\end{array}$ & $91.5 \%$ & $91.3 \%$ & 0.971 & $97.8 \%$ & $98.2 \%$ & 0.002 & $52.4 \%$ & $88.6 \%$ & 0.001 & $98.0 \%$ & $75.6 \%$ & $70.6 \%$ & 0.001 \\
\hline $\begin{array}{c}\text { Do you know about } \\
\text { Nuremburg code? } \\
\text { Yes } \\
\end{array}$ & $45.2 \%$ & $70.0 \%$ & 0.899 & $98.4 \%$ & $98.2 \%$ & 0.040 & $91.7 \%$ & $41.8 \%$ & 0.001 & $96.7 \%$ & $94.7 \%$ & $82.4 \%$ & 0.001 \\
\hline $\begin{array}{c}\text { Do you know about ICMR } 2017 \\
\text { guidelines? } \\
\text { Yes } \\
\end{array}$ & $19.9 \%$ & $23.1 \%$ & 0.506 & $90.6 \%$ & $98.2 \%$ & 0.011 & $85.7 \%$ & $57.1 \%$ & 0.024 & $96.7 \%$ & $92.7 \%$ & $84.4 \%$ & 0.001 \\
\hline $\begin{array}{c}\text { Do you know about } 2008 \\
\text { Helsinki Declaration? } \\
\text { Yes }\end{array}$ & $97.6 \%$ & $98.1 \%$ & 0.768 & $83.4 \%$ & $98.2 \%$ & 0.001 & $41.9 \%$ & $58.1 \%$ & 0.017 & $96.7 \%$ & $93.7 \%$ & $85.4 \%$ & 0.087 \\
\hline $\begin{array}{c}\text { Do you entertain patients' } \\
\text { questions during their visits? } \\
\text { Yes }\end{array}$ & $20.3 \%$ & $21.2 \%$ & 0.861 & $90.6 \%$ & $98.2 \%$ & 0.032 & $40.8 \%$ & $59.7 \%$ & 0.005 & $96.7 \%$ & $95 \cdot 7 \%$ & $86.4 \%$ & 0.087 \\
\hline $\begin{array}{c}\text { Have you attended training in } \\
\text { bio ethics? } \\
\text { Yes } \\
\end{array}$ & $48.0 \%$ & $49.0 \%$ & 0.855 & $83.4 \%$ & $98.2 \%$ & 0.029 & $40.3 \%$ & $59.7 \%$ & 0.002 & $96.7 \%$ & $94.7 \%$ & $87.4 \%$ & 0.001 \\
\hline $\begin{array}{c}\text { Is there an ethical committee } \\
\text { in your institution? } \\
\text { Yes }\end{array}$ & $73.6 \%$ & $75.6 \%$ & 0.280 & $97.5 \%$ & $98.2 \%$ & 0.004 & $72.2 \%$ & $27.8 \%$ & 0.001 & $96.7 \%$ & $95 \cdot 7 \%$ & $88.4 \%$ & 0.001 \\
\hline $\begin{array}{c}\text { How important is knowledge } \\
\text { of ethics to you in your work? } \\
\text { Very } \\
\text { Moderately } \\
\text { A little } \\
\text { Not at all }\end{array}$ & $\begin{array}{c}1.6 \% \\
1.6 \% \\
46.3 \% \\
2.4 \%\end{array}$ & $\begin{array}{c}1.3 \% \\
1.9 \% \\
49.0 \% \\
28.5 \%\end{array}$ & 0.983 & $\begin{array}{c}2.1 \% \\
1.1 \% \\
95.0 \% \\
1.8 \%\end{array}$ & $\begin{array}{l}98.2 \% \\
0.2 \% \\
1.2 \% \\
0.2 \%\end{array}$ & 0.001 & $\begin{array}{l}32.2 \% \\
39.7 \% \\
19.2 \% \\
10.2 \%\end{array}$ & $\begin{array}{c}60.3 \% \\
22.1 \% \\
14.2 \% \\
4.2 \%\end{array}$ & 0.025 & $96.7 \%$ & $92.7 \%$ & $87.4 \%$ & 0.001 \\
\hline $\begin{array}{c}\text { How often do you come across } \\
\text { any ethical issues? } \\
\text { Daily } \\
\text { Weekly } \\
\text { Monthly } \\
\text { Yearly } \\
\text { Never }\end{array}$ & $\begin{array}{c}0.3 \% \\
0.3 \% \\
0.3 \% \\
49.2 \% \\
42.3 \%\end{array}$ & $\begin{array}{c}0.6 \% \\
0.4 \% \\
5.5 \% \\
48.2 \% \\
43.3 \%\end{array}$ & 0.674 & $\begin{array}{c}92.2 \% \\
2.1 \% \\
1.1 \% \\
2.1 \% \\
1.1 \%\end{array}$ & $\begin{array}{c}98.2 \% \\
0.2 \% \\
1.2 \% \\
0.2 \% \\
1.5 \%\end{array}$ & 0.04 & $\begin{array}{c}4.4 \% \\
4.4 \% \\
11.2 \% \\
68.8 \% \\
11.4 \%\end{array}$ & $\begin{array}{l}39.7 \% \\
19.2 \% \\
10.2 \% \\
31.3 \% \\
0.6 \%\end{array}$ & 0.004 & $96.7 \%$ & $91.7 \%$ & $89.4 \%$ & 0.001 \\
\hline
\end{tabular}




\begin{tabular}{|c|c|c|c|c|c|c|c|c|c|c|c|c|c|}
\hline $\begin{array}{l}\text { How often do patients ask you } \\
\text { about their diagnosis? } \\
\text { Never } \\
\text { Seldom } \\
\text { Always }\end{array}$ & $\begin{array}{l}0.4 \% \\
45.1 \% \\
54.5 \%\end{array}$ & $\begin{array}{c}0.6 \% \\
45.2 \% \\
54.8 \%\end{array}$ & 0.809 & $\begin{array}{c}3.2 \% \\
13.2 \% \\
85.9 \%\end{array}$ & $\begin{array}{c}0.2 \% \\
1.2 \% \\
98.2 \%\end{array}$ & 0.006 & $\begin{array}{c}4.2 \% \\
34.4 \% \\
61.5 \%\end{array}$ & $\begin{array}{c}4.2 \% \\
61.5 \% \\
38.5 \%\end{array}$ & 0.164 & $96.7 \%$ & $92.7 \%$ & $90.4 \%$ & 0.001 \\
\hline $\begin{array}{c}\text { How often do you discuss your } \\
\text { daily cases with your } \\
\text { colleagues? } \\
\text { Seldom } \\
\text { Always } \\
\end{array}$ & $\begin{array}{l}12.2 \% \\
87.8 \%\end{array}$ & $\begin{array}{l}8.3 \% \\
91.3 \%\end{array}$ & 0.394 & $\begin{array}{l}15.2 \% \\
85.5 \%\end{array}$ & $\begin{array}{c}2.2 \% \\
98.2 \%\end{array}$ & 0.015 & $\begin{array}{l}45.8 \% \\
54.5 \%\end{array}$ & $\begin{array}{l}54.8 \% \\
45.5 \%\end{array}$ & 0.027 & $96.7 \%$ & $91.7 \%$ & $85.4 \%$ & 0.001 \\
\hline $\begin{array}{l}\text { How did you acquire your } \\
\text { knowledge of bioethics? } \\
\text { During training } \\
\text { Experience at work } \\
\text { Lectures/Seminar } \\
\text { One's own reading } \\
\text { Others (internet, court } \\
\text { reports, newspapers, etc) }\end{array}$ & $\begin{array}{c}0.4 \% \\
44.1 \% \\
1.1 \% \\
6.2 \% \\
48.2 \%\end{array}$ & $\begin{array}{c}0.01 \% \\
54.2 \% \\
0.4 \% \\
1.9 \% \\
45.2 \%\end{array}$ & 0.438 & $\begin{array}{l}92.2 \% \\
7.4 \% \\
1.2 \% \\
0.2 \% \\
1.2 \%\end{array}$ & $\begin{array}{l}98.9 \% \\
0.2 \% \\
0.2 \% \\
0.1 \% \\
1.2 \%\end{array}$ & 0.024 & $\begin{array}{l}41.9 \% \\
30.2 \% \\
24.5 \% \\
13.2 \% \\
1.2 \%\end{array}$ & $\begin{array}{c}58.9 \% \\
31.2 \% \\
19.2 \% \\
10.2 \% \\
0.2 \%\end{array}$ & 0.073 & $96.7 \%$ & $92.7 \%$ & $91.4 \%$ & 0.001 \\
\hline $\begin{array}{c}\text { Have you taken informed } \\
\text { consent from patients? } \\
\text { No } \\
\text { Verbal } \\
\text { Signed }\end{array}$ & $\begin{array}{c}0.1 \% \\
44 \cdot 7 \% \\
55 \cdot 3 \%\end{array}$ & $\begin{array}{c}0.2 \% \\
44.2 \% \\
55.8 \%\end{array}$ & 0.877 & $\begin{array}{c}5.5 \% \\
13.4 \% \\
84.7 \%\end{array}$ & $\begin{array}{c}0.2 \% \\
3.4 \% \\
97.9 \%\end{array}$ & 0.031 & $\begin{array}{l}24.5 \% \\
13.2 \% \\
39.6 \%\end{array}$ & $\begin{array}{l}24.5 \% \\
13.2 \% \\
60.4 \%\end{array}$ & 0.001 & $96.7 \%$ & $93.7 \%$ & $89.4 \%$ & 0.001 \\
\hline $\begin{array}{c}\text { Does your institute have } \\
\text { separate committees for } \\
\text { reviewing animal and human } \\
\text { research projects? } \\
\text { Yes } \\
\text { No } \\
\text { Not sure } \\
\end{array}$ & $\begin{array}{l}98.2 \% \\
0.4 \% \\
1.4 \%\end{array}$ & $\begin{array}{c}97.8 \% \\
1.2 \% \\
1.0 \%\end{array}$ & 0.804 & $\begin{array}{c}2.2 \% \\
4.2 \% \\
94.4 \%\end{array}$ & $\begin{array}{c}1.2 \% \\
3.2 \% \\
96.6 \%\end{array}$ & 0.027 & $\begin{array}{c}13.8 \% \\
3.2 \% \\
75.7 \%\end{array}$ & $\begin{array}{l}13.8 \% \\
24.3 \% \\
61.2 \%\end{array}$ & 0.064 & $96.7 \%$ & $94 \cdot 7 \%$ & $85.4 \%$ & 0.001 \\
\hline \multicolumn{14}{|c|}{ ATTITUDE } \\
\hline $\begin{array}{c}\text { Doctors know the best } \\
\text { irrespective of patients' } \\
\text { opinion } \\
\text { Slightly agree } \\
\text { Agree } \\
\text { Slightly disagree } \\
\text { Disagree } \\
\text { Not sure }\end{array}$ & $\begin{array}{l}38.2 \% \\
21.5 \% \\
39.8 \% \\
0.4 \% \\
0.1 \%\end{array}$ & $\begin{array}{c}24.2 \% \\
38.5 \% \\
15.2 \% \\
8.1 \% \\
14.0 \%\end{array}$ & 0.532 & $\begin{array}{c}41.6 \% \\
15.0 \% \\
3.1 \% \\
3.2 \% \\
31.7\end{array}$ & $\begin{array}{c}37.8 \% \\
15.0 \% \\
3.1 \% \\
3.2 \% \\
31.7\end{array}$ & 0.100 & $\begin{array}{c}73.6 \% \\
13.8 \% \\
8.6 \% \\
3.2 \% \\
0.8 \%\end{array}$ & $\begin{array}{c}38.3 \% \\
13.8 \% \\
71.5 \% \\
8.6 \% \\
3.2 \%\end{array}$ & 0.001 & $96.7 \%$ & $96.7 \%$ & $91.4 \%$ & 0.001 \\
\hline
\end{tabular}




\begin{tabular}{|c|c|c|c|c|c|c|c|c|c|c|c|c|c|}
\hline $\begin{array}{c}\text { Patient should always be } \\
\text { informed of wrongdoing } \\
\text { Slightly agree } \\
\text { Agree } \\
\text { Slightly disagree } \\
\text { Disagree } \\
\text { Not sure }\end{array}$ & $\begin{array}{c}24.2 \% \\
26.0 \% \\
15.4 \% \\
15.5 \% \\
18.9 \%\end{array}$ & $\begin{array}{l}24.0 \% \\
26.0 \% \\
17.8 \% \\
15.6 \% \\
16.6 \%\end{array}$ & 0.547 & $\begin{array}{c}46.9 \% \\
15.0 \% \\
3.2 \% \\
31.7 \\
3.2 \%\end{array}$ & $\begin{array}{c}48.4 \% \\
16.1 \% \\
13.1 \% \\
3.2 \% \\
19.2 \%\end{array}$ & 0.882 & $\begin{array}{c}0.3 \% \\
88.6 \% \\
8.6 \% \\
1.2 \% \\
0.2 \%\end{array}$ & $\begin{array}{c}0.2 \% \\
95.9 \% \\
1.2 \% \\
0.2 \% \\
3.0 \%\end{array}$ & 0.029 & $96.7 \%$ & $96.7 \%$ & $90.4 \%$ & 0.001 \\
\hline $\begin{array}{c}\text { Patients' wishes should always } \\
\text { be adhered to } \\
\text { Slightly agree } \\
\text { Agree } \\
\text { Slightly disagree } \\
\text { Disagree } \\
\text { Not sure } \\
\end{array}$ & $\begin{array}{c}24.3 \% \\
67.1 \% \\
5.3 \% \\
3.2 \% \\
1.2 \%\end{array}$ & $\begin{array}{l}5.3 \% \\
3.2 \% \\
1.2 \% \\
67.3 \% \\
24.3 \%\end{array}$ & 0.916 & $\begin{array}{c}33.4 \% \\
15.0 \% \\
3.1 \% \\
3.2 \% \\
31.7\end{array}$ & $\begin{array}{c}49.3 \% \\
15.0 \% \\
3.1 \% \\
3.2 \% \\
31.7\end{array}$ & 0.624 & $\begin{array}{c}87.0 \% \\
8.6 \% \\
1.2 \% \\
0.2 \% \\
0.1 \%\end{array}$ & $94 \cdot 3 \%$ & 0.034 & $96.7 \%$ & $96.7 \%$ & $89.4 \%$ & 0.001 \\
\hline $\begin{array}{c}\text { Confidentiality cannot be } \\
\text { maintained in modern care } \\
\text { and should be abandoned } \\
\text { Slightly agree } \\
\text { Agree } \\
\text { Slightly disagree } \\
\text { Disagree } \\
\text { Not sure } \\
\end{array}$ & $\begin{array}{c}26.8 \% \\
24.2 \% \\
23.1 \% \\
18.2 \% \\
8.1 \%\end{array}$ & $\begin{array}{c}3.1 \% \\
3.2 \% \\
31.7 \% \\
62.2 \% \\
\mathbf{2 . 2} \%\end{array}$ & $0.35^{2}$ & $\begin{array}{c}56.6 \% \\
15.0 \% \\
3.1 \% \\
3.2 \% \\
31.7\end{array}$ & $\begin{array}{c}62.2 \% \\
15.0 \% \\
3.1 \% \\
3.2 \% \\
31.7\end{array}$ & 0.754 & $\begin{array}{c}8.6 \% \\
1.2 \% \\
0.2 \% \\
0.1 \% \\
81.3 \%\end{array}$ & $\begin{array}{c}1.2 \% \\
0.2 \% \\
0.1 \% \\
2.2 \% \\
94.3 \%\end{array}$ & 0.001 & $96.7 \%$ & $96.7 \%$ & $88.4 \%$ & 0.001 \\
\hline $\begin{array}{l}\text { Consent is required only in } \\
\text { case of operations and not for } \\
\text { tests and medications } \\
\text { Slightly agree } \\
\text { Agree } \\
\text { Slightly disagree } \\
\text { Disagree } \\
\text { Not sure } \\
\end{array}$ & $\begin{array}{c}23.1 \% \\
18.0 \% \\
7.2 \% \% \\
24.2 \% \\
27.5 \%\end{array}$ & $\begin{array}{c}3.1 \% \\
3.2 \% \\
31.7 \% \\
2.2 \% \\
32.7 \%\end{array}$ & 0.082 & $\begin{array}{c}45.5 \% \\
15.0 \% \\
3.1 \% \\
3.2 \% \\
31.7\end{array}$ & $\begin{array}{c}52.2 \% \\
15.0 \% \\
3.1 \% \\
3.2 \% \\
31.7\end{array}$ & 0.688 & $\begin{array}{c}8.6 \% \\
1.2 \% \\
0.2 \% \\
82.9 \% \\
0.1 \%\end{array}$ & $\begin{array}{c}0.2 \% \\
1.2 \% \\
0.2 \% \\
2.2 \% \\
96.2 \%\end{array}$ & 0.001 & $96.7 \%$ & $96.7 \%$ & $86.4 \%$ & 0.001 \\
\hline $\begin{array}{l}\text { Certain medical practitioners } \\
\text { charge more from rich } \\
\text { patients to compensate for } \\
\text { treating the poor Do you } \\
\text { agree with this? } \\
\text { Slightly agree } \\
\text { Agree } \\
\text { Slightly disagree } \\
\text { Disagree } \\
\text { Not sure }\end{array}$ & $\begin{array}{c}37.3 \% \\
23.1 \% \\
18.2 \% \\
8.1 \% \\
24.0 \%\end{array}$ & $\begin{array}{c}41.3 \% \\
3.1 \% \\
3.2 \% \\
31.7 \% \\
2.2 \%\end{array}$ & 0.023 & $\begin{array}{c}15.0 \% \\
3.1 \% \\
3.2 \% \\
31.7 \\
45.5 \%\end{array}$ & $\begin{array}{c}15.0 \% \\
3.1 \% \\
3.2 \% \\
31.7 \\
52.2 \%\end{array}$ & 0.966 & $\begin{array}{c}8.6 \% \\
1.2 \% \\
0.2 \% \\
0.1 \% \\
79.7 \%\end{array}$ & $\begin{array}{c}1.2 \% \\
0.2 \% \\
2.2 \% \\
2.1 \% \\
94.3 \%\end{array}$ & 0.001 & $96.7 \%$ & $96.7 \%$ & $85.4 \%$ & 0.001 \\
\hline
\end{tabular}




\begin{tabular}{|c|c|c|c|c|c|c|c|c|c|c|c|c|c|}
\hline $\begin{array}{c}\text { Ethical conduct is important } \\
\text { only for avoiding legal action } \\
\text { Slightly agree } \\
\text { Agree } \\
\text { Slightly disagree } \\
\text { Disagree } \\
\text { Not sure } \\
\end{array}$ & $\begin{array}{c}25.0 \% \\
13.1 \% \\
13.2 \% \\
31.7 \% \\
17.0 \%\end{array}$ & $\begin{array}{l}13.5 \% \\
3.1 \% \\
3.2 \% \\
31.7 \% \\
2.2 \%\end{array}$ & 0.617 & $\begin{array}{l}73.8 \% \\
15.0 \% \\
3.1 \% \\
3.2 \% \\
31.7\end{array}$ & $\begin{array}{c}75 \cdot 5 \% \\
15.0 \% \\
3.1 \% \\
3.2 \% \\
31.7\end{array}$ & 0.276 & $\begin{array}{c}77.2 \% \\
1.2 \% \\
0.2 \% \\
2.2 \% \\
12.1 \%\end{array}$ & $\begin{array}{l}91.7 \% \\
1.2 \% \\
0.2 \% \\
4.7 \% \\
2.2 \%\end{array}$ & 0.001 & $96.7 \%$ & $96.7 \%$ & $89.4 \%$ & 0.001 \\
\hline $\begin{array}{c}\text { Child should never be treated } \\
\text { without concern of the } \\
\text { parents } \\
\text { Slightly agree } \\
\text { Agree } \\
\text { Slightly disagree } \\
\text { Disagree } \\
\text { Not sure }\end{array}$ & $\begin{array}{c}15.0 \% \\
23.1 \% \\
37 \cdot 3 \% \\
18.2 \% \\
8.1 \%\end{array}$ & $\begin{array}{l}13.5 \% \\
23.1 \% \\
47 \cdot 3 \% \\
18.2 \% \\
8.1 \%\end{array}$ & 0.713 & $\begin{array}{c}52.2 \% \\
15.0 \% \\
3.1 \% \\
3.2 \% \\
31.7\end{array}$ & $\begin{array}{c}56.7 \% \\
15.0 \% \\
3.1 \% \\
3.2 \% \\
31.7\end{array}$ & 0.708 & $\begin{array}{c}74.8 \% \\
1.2 \% \\
0.2 \% \\
2.2 \% \\
12.1 \%\end{array}$ & $\begin{array}{c}60.9 \% \\
1.2 \% \\
0.2 \% \\
2.2 \% \\
12.1 \%\end{array}$ & 0.008 & $96.7 \%$ & $96.7 \%$ & $92.4 \%$ & 0.001 \\
\hline $\begin{array}{c}\text { Do you have any clinical } \\
\text { ethics or research ethics class? } \\
\text { Yes } \\
\text { No }\end{array}$ & $\begin{array}{l}19.4 \% \\
80.6 \%\end{array}$ & $\begin{array}{l}18.3 \% \\
81.7 \%\end{array}$ & 0.654 & $\begin{array}{l}52.2 \% \\
42.9 \%\end{array}$ & $\begin{array}{l}73.2 \% \\
27.2 \%\end{array}$ & 0.932 & $\begin{array}{c}68.3 \% \\
31.7 \%\end{array}$ & $\begin{array}{l}71.3 \% \\
28.7 \%\end{array}$ & 0.001 & $96.7 \%$ & $96.7 \%$ & $92.4 \%$ & 0.001 \\
\hline $\begin{array}{l}\text { If so, how many hours or } \\
\text { classes } \\
\text { One hour/3classes/month } \\
\text { Two hours/ } 2 \text { classes/month } \\
30 \text { minutes/ } 1 \text { class/month }\end{array}$ & $\begin{array}{c}3.2 \% \\
5.3 \% \\
90.2 \%\end{array}$ & $\begin{array}{c}2.2 \% \\
6.4 \% \\
(91.3 \%)\end{array}$ & 0.735 & $\begin{array}{l}40.8 \% \\
20.2 \% \\
42.2 \%\end{array}$ & $\begin{array}{r}8.2 \% \\
12.2 \% \\
79.5 \%\end{array}$ & $82.2 \%$ & $\begin{array}{r}11.1 \% \\
12.2 \% \\
77.2 \%\end{array}$ & $\begin{array}{l}5.5 \% \\
91.7 \% \\
2.8 \%\end{array}$ & 0.581 & $96.7 \%$ & $96.7 \%$ & $92.4 \%$ & 0.408 \\
\hline \multicolumn{14}{|c|}{ PRACTICE } \\
\hline $\begin{array}{l}\text { When people holding certain } \\
\text { religious beliefs refuse to take } \\
\text { blood, undergo surgery or } \\
\text { accept treatment, what is your } \\
\text { stand? } \\
\text { Respect the patient's decision } \\
\text { Try to perform the procedure } \\
\text { forcibly } \\
\text { Refer to a doctor who shares } \\
\text { the patient's beliefs } \\
\text { Any other (specify) }\end{array}$ & $\begin{array}{l}0.8 \% \\
18.3 \% \\
65.9 \% \\
0.8 \%\end{array}$ & $\begin{array}{l}0.9 \% \\
5.8 \% \\
68.3 \% \\
20.2 \%\end{array}$ & 0.002 & $\begin{array}{l}6.3 \% \\
1.6 \% \\
87.8 \% \\
4.4 \%\end{array}$ & $\begin{array}{l}0.8 \% \\
0.7 \% \\
92.0 \% \\
6.1 \%\end{array}$ & 0.122 & $\begin{array}{l}13.8 \% \\
71.5 \% \\
8.6 \% \\
3.2 \%\end{array}$ & $\begin{array}{l}5.5 \% \\
90.4 \% \\
2.2 \% \\
2.2 \%\end{array}$ & 0.002 & $\begin{array}{l}0.0 \% \\
0.0 \% \\
96.7 \% \\
0.0 \%\end{array}$ & $\begin{array}{l}0.0 \% \\
0.0 \% \\
96.7 \% \\
0.0 \%\end{array}$ & $\begin{array}{l}0.0 \% \\
0.0 \% \\
92.7 \% \\
0.0 \%\end{array}$ & 0.001 \\
\hline
\end{tabular}




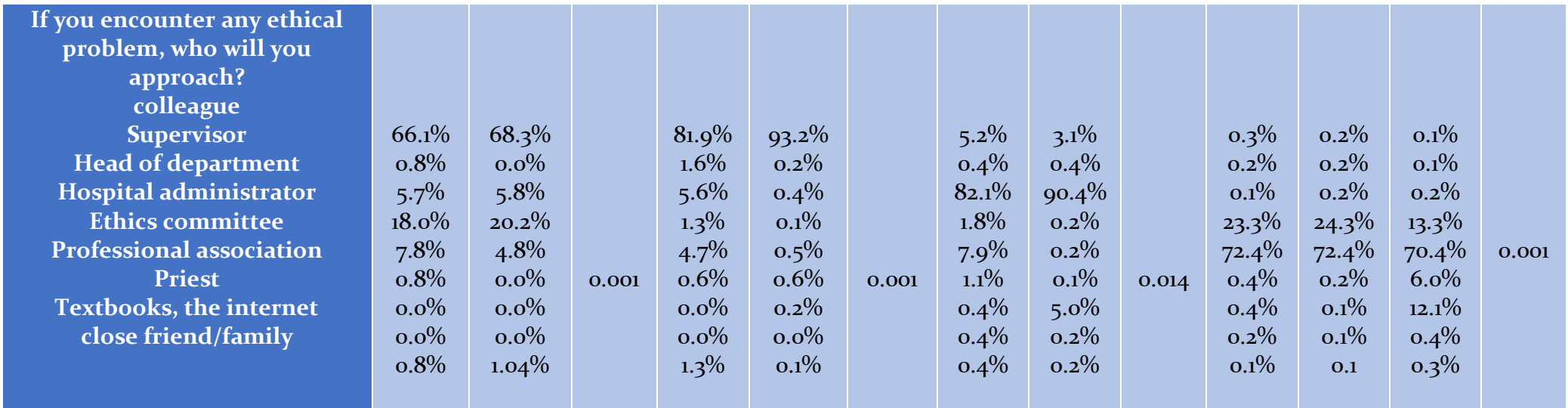

Table 2. Knowledge, Attitude and Practice of Health Care Ethics in the study population (DI: Dental College Interns, DP: Dental College postgraduates, MCI: Medical College Interns, MCPG: Medical College postgraduates, NTY: Nursing College Third Year, NFY: Nursing College Final Year, MF: Medical Faculty, DF: Dental Faculty, NF: Nursing Faculty, pV: p value)

declaration $(\mathrm{p}<0.01)$ and there was no significant difference between the attitude towards the information of wrong-doing to the patients. There was a significant difference in the practice of health care ethics and most of the medical postgraduates approached the concerned the head of the department for any ethical problems ( $p>0.001)$.

Among nursing college third years and final years, knowledge regarding Hippocratic oath ( $>0.01)$; Nuremberg code ( $p>0.001)$; ICMR guidelines (p>0.024) and Helsinki declaration ( $p>0.017$ ) had a significant difference. There was no significant difference between knowledge regarding asking the patients for diagnosis and regarding the animal and human research ethical committee $(\mathrm{p}<0.0064)$. There was a significant difference between the attitude and practice of health care ethics ( $>>0.001$ ) and the approach of the hospital administrator for any ethical committee problems ( $p>0.014)$

Among the faculty of medical; dental and nursing colleges, it was observed that medical faculty and dental faculty had better knowledge regarding the Hippocratic oath; Nuremberg code; ICMR guidelines and Helsinki declaration regarding the attitude and practice most of the dental and medical faculty has a significant difference. 
Figure 1 describes the health care ethics that most of the medical post graduates would practice $(\mathrm{p}<0.001)$;most of dental postgraduates and interns $(\mathrm{p}<0.002)$ would practice with the patient beliefs with reference to certain doctor whom belief. The nursing students would like to practice the procedure forcibly $(\mathrm{p}<0.002)$.

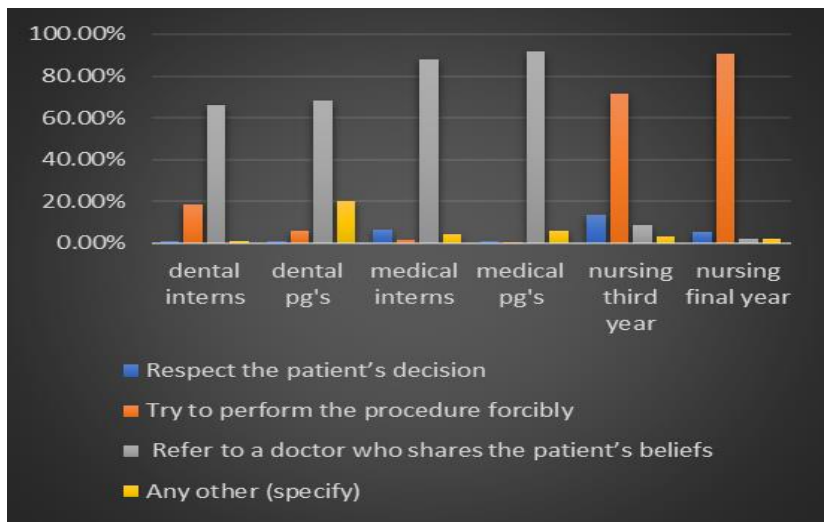

Figure 1. Practice of Health Care Ethics Patient's Holding Religious Beliefs among Dental, Medical and Nursing Interns and Post Graduates

Figure 2 describes practice of health care ethics most of the medical post graduates approach to the concerned the head of the department for any ethical problems ( $>>0.001)$; practice of dental postgraduates with comparison of dental interns ( $>>0.02)$ and most of dental postgraduates and interns have a practice of approaching the colleges for health care problem. and practice of health care ethics ( $>0.001)$ and the approach the hospital administrator for any ethical committee problems ( $\mathrm{p}>0.014)$.

Figure 3 describes the health care ethics that the medical, dental and nursing college faculty would like to practice the patient's belief with reference to the certain doctor whom belief.

Figure 4 represents the health care ethics that the medical, dental and nursing college faculty would like to approach the ethical committee head when they encounter any ethical dilemmas.

\section{DISCUSSION}

Health Care-ethics is a division of bioethics which deals with the ethical dimensions of day-to-day patient care. The attitudes towards informed consent, truth-telling, confidentiality, abortion, euthanasia, and treating a non-compliant patient are aspects of care ethics that may well be influenced by the social, cultural and religious background, and the gender of the healthcare. provider. ${ }^{8}$

The findings of the present study show that the medical interns and postgraduates' knowledge show the significant difference between the Hippocratic oath, Nuremberg code, ICMR guidelines, and Helsinki declaration and dental interns and graduates shows no significant difference which is relevant to the study done by Adhikari et al. ${ }^{9}$ The knowledge of third years nursing students have lower experience compared with the final year students. Regarding the attitude of medical interns and postgraduates, there is a significant difference in some aspects such as the informing of the patient wrongdoing; consent requires for the medical investigations and confidentiality of the patient while dental interns and postgraduates do not have a significant difference between the attitude which is parallel to the study done by Anup et al. (2014) $)^{5}$ and Seetharaman et al. (2006) ${ }^{10}$ while there is no significant difference between the attitude of third and final year nursing college students. Lack of internship in the Nursing curriculum and absence of postgraduates in the selected colleges, the present study included only the third and final years. The present study says that the practice of health care ethics among medical interns and postgraduates has significant differences, and dental interns and graduates show a considerable difference.

Comparison of the medical; dental and nursing faculty shows no significant difference between the knowledge and attitude. But in some aspects regarding the health care ethics such as that the ICMR guidelines; Helsinki declaration there is little more knowledge to the medical faculty compared with the dental and nursing faculty. The problems encountered through health care ethics were more in the medical and dental faculty than in the nursing faculty. The practice also shows no significant difference between the medical and dental and nursing faculty. It also shows the close relationship between attitude and increases in age and work experience, which show similar findings conducted by Chopra M. et al. 2013 and Brogen SA in 2009.112

The knowledge and attitude of the postgraduates found to be more compared to undergraduates.

Limitations: As the study is questionnaire-based, the participants' responses may change after some time, which means the perception might change when the questionnaire administered for the second time. As it is 


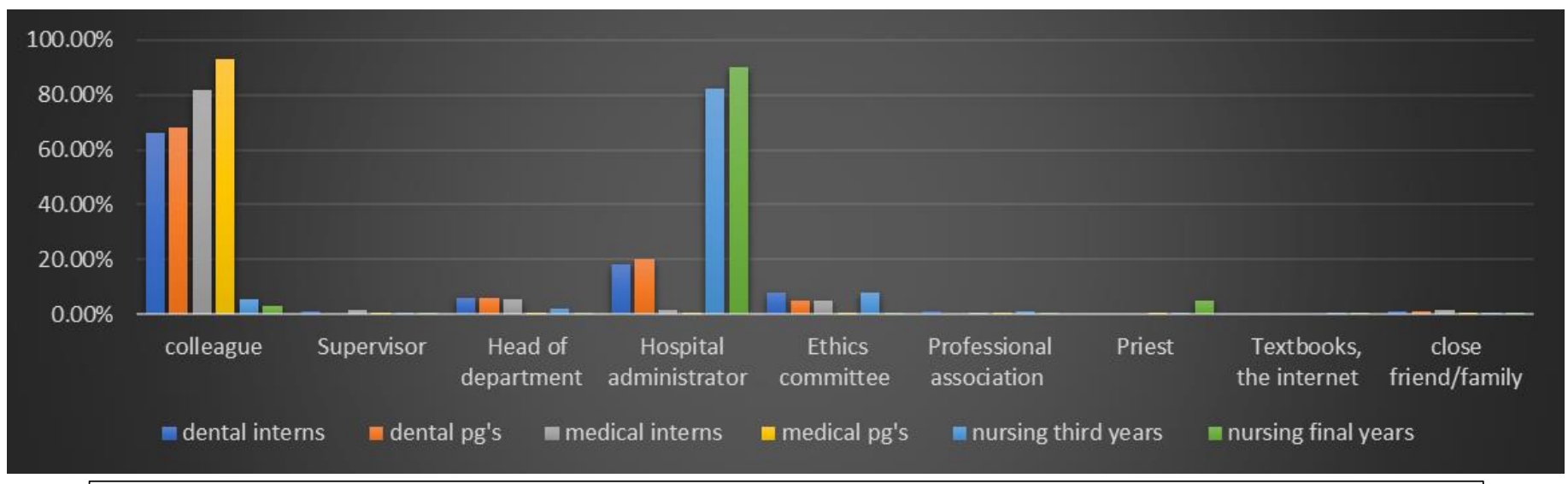

Figure 2. Practice of Health Care Ethics of Approaching the Ethical Dilemmas among Dental, Medical and Nursing Interns and Post Graduates



Figure 3. Practice of Health Care Ethics Patient's Holding Religious Beliefs among Dental, Medical and Nursing Faculty

a self-administrated questionnaire, there might be a chance of social desirability bias.

\section{CONCLUSION}

Health care ethics are one of the most neglected topics. The present study emphasizes that a significant proportion of the medical; dental and nursing students have aware of universally ethical principles of ethics, which are essential for clinical practice. However, some students feel difficulty in ethical dilemmas that are encounter.

Recommendations: The knowledge of healthcare ethics in curriculum and use of interactive techniques such as Seminars, Workshops, CME conferences would assist in bridging this gap to a certain extent at the undergraduate level and providing of journals, articles at the postgraduate level.

\section{REFERENCES}

1. Kesavan R, Mary AV, Priyanka M, Reashmi B. Knowledge of dental ethics and jurisprudence among dental practitioners in Chennai, India: A cross-sectional questionnaire study. J Oro Fac Sci. 2016;8:128-34.

2. Taruna D, Singh RNP, Suma BS, Mangal G. Dental Professionals Perspectives About the Consumer Protection Act and Ethical Practice in Patna, Bihar. European Journal of Pharmaceutical and Medical Research 2019:6(2):323-9.

3. Singh K, Shetty S, Bhat N, Sharda A, Agrawal A, Chaudhary H. Awareness of Consumer Protection Act among Doctors. J Dent (Tehran). 2010;7(1):19-23.

4. Gambhir RS, Dhaliwal JS, Anand S, Bhardwaj A. Knowledge and awareness of Consumer Protection Act among private dentists in Tricity, Punjab. J Family Med Prim Care. 2015; 4:347-51.

5. Anup N, Kumawat H, Biswas G, Pareek S, Tambi S. Knowledge, attitude \& practices regarding Ethics \& Law amongst medical and dental professionals in Rajasthan - A Questionnaire study. IOSR Journal of Dental and Medical Sciences (IOSR-JDMS) 2014;13(5): 102-9.

6. Mallela KK, Walia R, Devi CTM, Das M, Sepolia S, Sethi P. Knowledge, attitudes and practice about research ethics among dental faculty in the North India. J Int Oral Health. 2015;7(Suppl 2):52-6.

7. Janakiram C, Gardens SJ. Knowledge, attitudes and practices related to healthcare ethics among medical and dental postgraduate students in South India. Indian J Med Ethics. 2014; 11(2): 99-103. https://doi.org/10.20529/IJME.2014.025. 


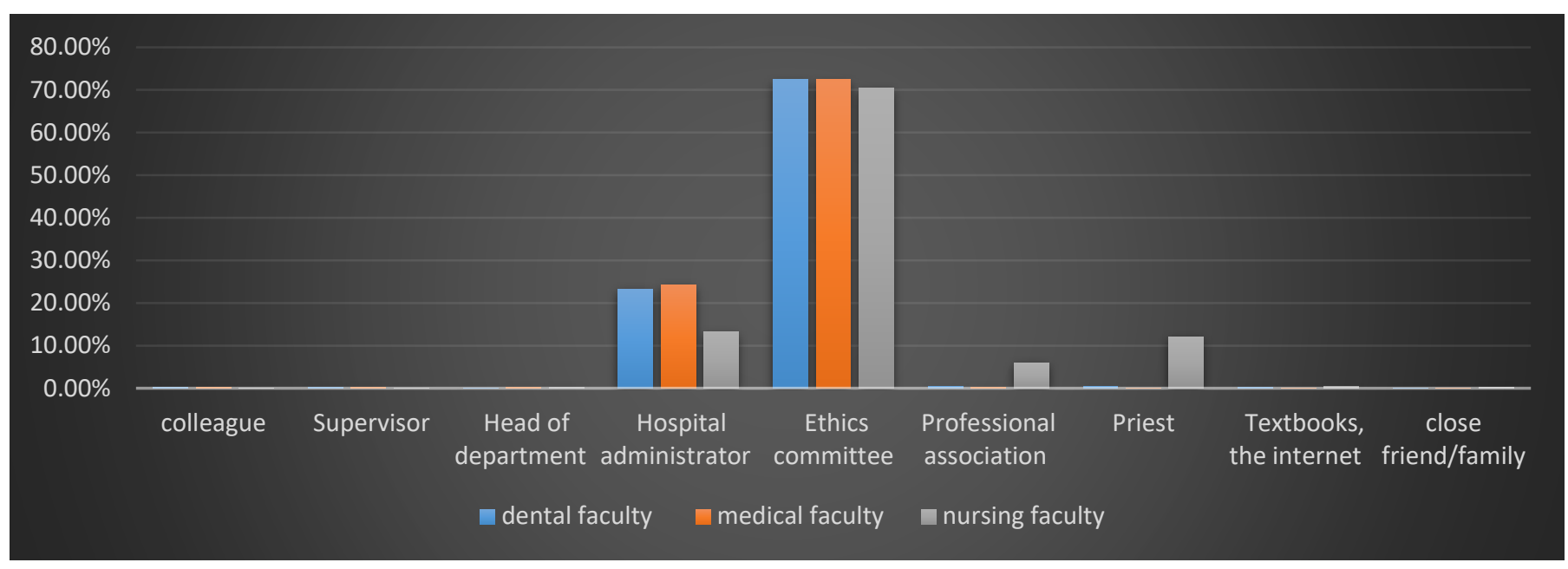

Figure 4. Practice of Health Care Ethics of Approaching the Ethical Dilemmas among Dental, Medical and Nursing Faculty

8. Hariharan S, Jonnalagadda R, Gora J. Knowledge, attitudes and practices of healthcare personnel towards Care-Ethics: A perspective from the Caribbean. The Internet Journal of Law, Healthcare and Ethics 2006;5(1):1-8.

9. Adhikari S, Paudel K, Aro AR, et al. Knowledge, attitude and practice of healthcare ethics among resident doctors and ward nurses from a resource poor setting, Nepal. BMC Med Ethics. 2016,17:68.

10. Hariharan S, Jonnalagadda R, Walrond E, Moseley $\mathrm{H}$. Knowledge, attitudes and practice of healthcare ethics and law among doctors and nurses in Barbados BMC Medical Ethics. 2006;7:7-16.
11. Chopra M, Bhardwaj A, Mithra P, Singh A, Siddiqui A, Rajesh DR. Current status of knowledge, attitudes and practices towards healthcare ethics among doctors and nurses from Northern India - A multicentre study. Journal of Krishna Institute of Medical Sciences University 2013;2(2):102-7.

12. Brogan SA, Rajkumari B, Laishram J, Joy A. Knowledge and attitude of doctors on medical ethics in a teaching hospital, Manipur. Indian J Med Ethics. 2009;6(4): 194-7.

Source of support: Nil, Conflict of interest: None declared

Cite this article as:

Pratyusha CH, Vineela P, Kumar SP, Nagarjuna P, Karishma J, Adithya P. Knowledge, Attitude and Practice of Health Care Ethics Among Medical, Dental and Nursing Colleges Students and Faculty in Visakhapatnam: A Questionnaire Study. Int Healthc Res J. 2020;4(5):126-135. https://doi.org/10.26440/IHRJ/0405.08283

\section{AUTHOR AFFILIATIONS: (*Corresponding Author)}

1. Post Graduate Student [ORCID: oooo-ooo1-6806-3401 (Dr. Pratyusha Chatti), ORCID: oooo-ooo1-9189-3075 (Dr. Karishma Janapareddy),

ORCID: 0000-0002-5720-9361 (Dr. Adithya Teja Prasad Pallekonda)]

2. Reader [ORCID: 0000-0003-3498-6325 (Dr. Vineela Parlapalli), ORCID: 0000-0002-8027-6597 (Dr. SivaKumar Pydi)]

3. Senior Lecturer (ORCID: 0ooo-0003-0541-5320)

Department of Public Health Dentistry, Anil Neerukonda Institute of Dental Sciences, Sangivalasa, Bheemunipatnam Mandal,

Visakhapatnam (Dt), Andhra Pradesh-531163 